\title{
Mobile Data Collector Routing Protocol Scheme for Scalable Dense Wireless Sensor Network to Optimize Node's Life
}

\author{
Farhan A. Siddiqui ${ }^{1}$, Jibran R. \\ Khan $^{2}$, Muhammad Saeed ${ }^{3}$ \\ Department of Computer Science - \\ UBIT, University of Karachi, \\ Pakistan
}

\author{
M. $\operatorname{Arshad}^{4}$ \\ Deanship of e-Learning and \\ Information Technology, Jazan \\ University, Jazan, Kingdom of Saudi \\ Arabia
}

\author{
Dr. Nasir Touheed ${ }^{5}$ \\ Institute of Business Administration \\ (IBA), University of Karachi, \\ Pakistan
}

\begin{abstract}
Wireless Sensor Networks (WSN) is a special kind of network communication architecture which has a very wide range of application and the cost-effectiveness of this architecture boosts its adaptability and usability. The erratic use of WSN, its rapid advancement has encouraged the research community to report several standing problems with WSN, among them is the concern of network life and node energy management in a dense network. This paper presents the experimental outcomes of using MDC multi-tier approach in dense network environments. Besides the node density, experiments also consider the interagricultural field hurdles that cause communication disturbance among the nodes that exist in ground level, or at some height above the farming field. The simulated experiment shows the noteworthy results, which comparatively enhance the network lifetime, efficiently utilizing individual node energy, and maximizing the content delivery.
\end{abstract}

Keywords-WSN; MDC; node density; energy efficient sensor networks; agriculture; robust networks

\section{INTRODUCTION}

During the last few years, WSN stands to be the prominent cost-effective solution of many prevailing challenges. The flexibility and adaptability of WSN rapidly capture a wide range of applications including business, industry, healthcare, and security. The continuous evolution of WSN, makes it compatible with traditional network architectures, interoperation ability, and compatibility[1]. Every technology, in its early age, possesses some holes and leaks that are identified with the passage of time. These loopholes depend on the nature of the architecture it is used in, traffic volume and velocity, and complexity of deployment. In the swift of WSN utilization among many problems, the core challenge that discourages affordability and deployment is to prolong the network lifespan in dense or complex network environment[2]-[4].

A WSN can be deployed as per the business and application requirement, either loosely or tightly coupled (normal to dense or highly complex) scenarios. The connectivity and communication among the deployed sensor nodes are supported by routing protocols[5]-[8]. Unlike traditional routing protocols, WSNs are much more flexible in such that they can be deployed in various formations such as fixed or mobile nodes where each individual node acts as a router and performs computation and operation to receive and send data to the corresponding node. Thus, the volume and communication traffic velocity of a node determine the life of the node.

Many techniques and routing protocols have been designed to minimize node interactivity to overcome the problem of battery diminution and maximize the life of a node which eventually prolongs the network life. These techniques are broadly categorized into two types of WSNs: Homogenous and Heterogeneous network. In Homogeneous WSN architecture, all participating nodes equally perform all tasks and act as a node to sense environmental data; and act as a router to transmit a packet from another node to the next hop, at the same time[8]-[11]. While in Heterogeneous WSN architecture, not all nodes are responsible to receive the environmental feeds. Instead, they are responsible to collect data from other nodes in its region and then deliver aggregated data to the upper hierarchical node of the same kind, these node are known as Head node or Cluster head [1], [5] . While other nodes, despite extensive communication with each other, kill themselves early and halt the network, they communicate with their master or head node to prevent energy depletion. In WSNs, it is also possible that some or all of the nodes are in motion or moveable, and the network can be of one of the two kinds discussed above: Homogeneous or Heterogeneous[12][15]. We have already discussed what factors influence the node energy consumption in a static or fixed sensor nodes environment. With node mobility, the energy utilization will also increase with respect to the frequency of node displacement, updating routing information, availability of path or node, the election of head node, and determining member nodes in a specific region and cluster environment. [16].

The problem becomes more complex and challenging when we talk about the dense network scenarios. The node density in a network elevates challenges of wireless sensors networks, such as routing information management, communication management, nodes' energy management (especially mobile node) etc. [17] These challenges make WSN a questionable choice for a cost-effective, prominent, and advanced solution. Also, some question its usability 
limitations i.e. where and in what environment WSN should be considered.

The rest of the paper is distributed as follows: The literature review discusses recent progress and approaches to diminish WSNs routing challenges; then we present our novel methodology along with result discussion with simulated outcomes which enhance network life; and then we finally summarize paper with a conclusion and future work discussion.

\section{RELATED WORK}

After Among all, there was one literature in previous study that specifically discusses the agriculture application need [18] of managing battery depletion which enriches the network lifecycle and impact of node density in agriculture fields. Based on the studied work, some prominent work taken as the reference point of problem and our prior knowledge and efforts in agro-economic applications of WSNs.

Salim et. al. in [19] used the LEACH protocol introducing an Intra-Balance technique which further advances the LEACH protocol by naming it IBLEACH. The idea works similarly to LEACH itself but differs in the communication scheme in which cluster head and their corresponding member nodes share the energy load based on various parameters that include remaining energy, energy required to transmit frame, and distance in each round. Besides $\mathrm{CH}$, aggregated cluster members list is also determined in each round which is responsible to send only one frame by an aggregator. The simulated environment resulted in empowering the network life comparative to LEACH measured performance and minor improvement as compared with other approaches derived from LEACH.

P. Varshney et. al. [20] measure the performance of two proactive routing protocols OLSR and STAR in a dense Adhoc environment. Their experiment was conducted with five different variations as $10,20,30,40$, and 50 nodes that gradually increased the number of nodes in a 500 square unit environment. The simulation was done on the QualNet, and results found that OLSR outperforms the STAR protocol in mobility scenarios. As the number of nodes increases with each iteration STAR's performance tends to go down and OLSR keep felicitating and increasing the network performance.

D. Elmazi et. al. [21] proposed the fussy-based system approach for a dense network to measure the performance in WSNs environment. This approach uses the wireless sensor actor network (WSAN) scheme. The author used two fuzzy based actor selection systems (FBASS) which were distinguished by the number of fussy rules. System 1 (FBASS1) used a comparatively low number of rules, while System 2 (FBASS2) had complex and high number of rules defined. The environment comprised of 3 elements:

1) Sense node

2) Actor node

3) Sink node.

The WSAN F system, when it transmits data calculates the distance between two actors each time and sends it to the nearer actor, and then the actor is responsible for delivering it to sink node. The actor selection in this scheme is based on a few things. These factors include the job type, distance, remaining energy which determines the selection probability of actor node (low, medium and high). Between the said two systems, the FBASS2 performed well due to the high number of predefined rules.

\section{ChallengeS}

With reference to study [18], it has been noticed that beyond the standing problems of network life, individual node energy and smart cluster head or actor selection are issues that need to be solved as well. The agriculture fields have another key obstacle in WSNs deployment which is communication among the ground sensor, above the field sensors, and sensors that are deployed at minimal or average height of field. The normal deployment troubled the agri-field hurdles and sensor data failed to be delivered at sink node. The problems that have been highlighted are:

- What if these dense sensors network is deployed in dense agriculture fields?

- How to keep the network performance high or consistent?

- How to prolong the network lifetime? And what technique should facilitate or overcome these issues?

\section{Simulation ENVIRONMENT}

The proposed solution is simulated under OPNET network modular environment to determine its worth and compare the results the previously presented result by using the same parameters of [18] listed in Table 1.

TABLE I. SimULATION PARAMETERS

\begin{tabular}{|l|l|}
\hline Parameters & \multicolumn{1}{|c|}{ Values } \\
\hline Number of nodes & 40 \\
\hline Simulation area & $1 \mathrm{~km}^{2}$ \\
\hline Transmission electronics $\left(\mathbf{E}_{\mathbf{T X}-\mathrm{elec}}\right)$ & $50 \mathrm{nj} / \mathrm{bit}$ \\
\hline Receiver electronics $\left(\mathbf{E}_{\mathbf{R X}-\mathrm{elec}}\right)$ & \\
\hline Transmit amplifier (Eamp) & $100 \mathrm{pj} / \mathrm{bit} / \mathrm{m}^{2}$ \\
\hline Node energy & $2 \mathrm{joules}$ \\
\hline Number of MDCs & 2 \\
\hline MDC beacon message rate & $5 \mathrm{~s}$ \\
\hline MDC velocity & $0.054 \mathrm{~m} / \mathrm{s}$ \\
\hline MDC energy & $30 \mathrm{joules}$ \\
\hline Packet size & $160 \mathrm{bits} / \mathrm{packet}$ \\
\hline
\end{tabular}


In this experimental work, number of 40 nodes used that randomly deployed in $1 \mathrm{~km} 2$ area. Where, each sensor node (N) has the limited energy of 2 joules and MDC that is mobile in nature has 30 joules, which assure that MDC should not die before the sensor node. MDC displacement velocity is set as $0.054 \mathrm{~m} / \mathrm{s}$. MDC speed keep relatively slow which allows enough time to bi-directional communication between $\mathrm{CH}$ MDC and MDC-BS, and the size of each data packet is consider as 160 bits/packet.

\section{RESULTS AND DISCUSSION}

In our previous work [18] we have presented the new methodology and communication approach which significantly outperform the LEACH and other derived techniques by utilizing the hybrid multi-tier Mobile Data Collector (MDC) approach. The basic concept of MDC architecture shown in Fig.1.

Fig. 1 shows the sample agricultural or farm field with obstacles that could lead to inter-cluster and inter-node communication and data delivery issues. Where basic functionality of each participating element is as follows; NDCs are node data collectors which are static or fixed nodes that sense the environmental data and store it, CDCs are the cluster data collectors which are responsible for collecting the data from the NDCs periodically, MDCs are the mobile data collectors which, as per their name, are mobile in nature and can be displaced from one point to another in the region and these MDCs predesignated to get aggregated data from CDCs in its region. Whereas SDC is sink data collector point which is the destination point of sensed environmental data that is attached with the application to measure and store the information. CR represents the cluster region which specifies the nodes' associated membership with CDC in a logical region. Field Area (FA) denotes the experimental area of an open farming land. DOPs are the data obstacle points which cause communication disturbance among the NDCs, CDCs, and MDCs.

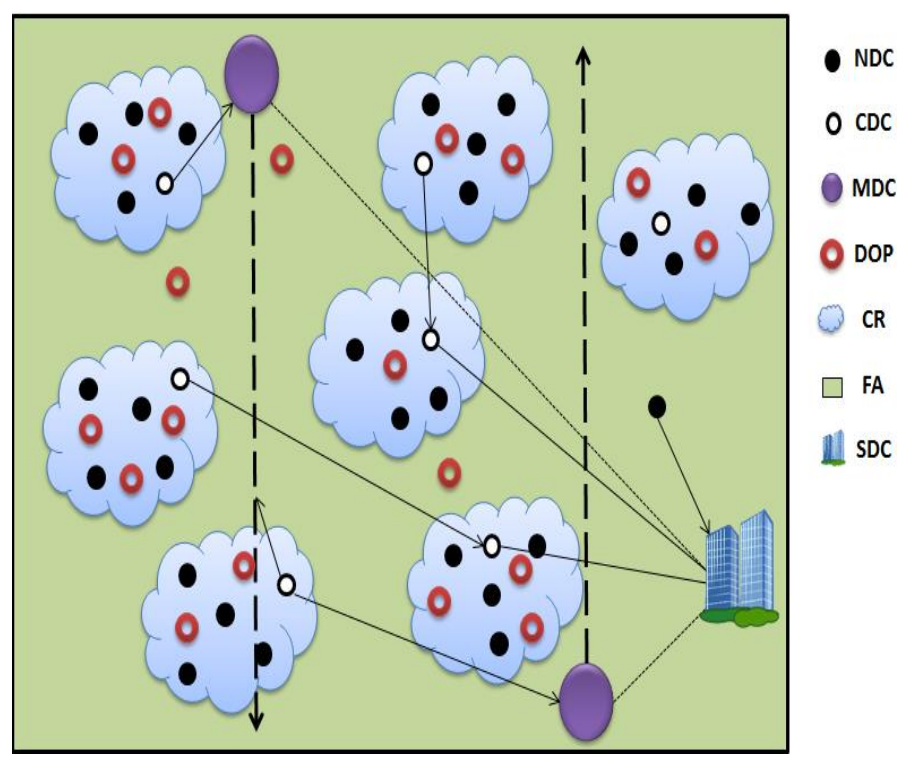

Fig. 1. Multi-Tier MDC Architecture.

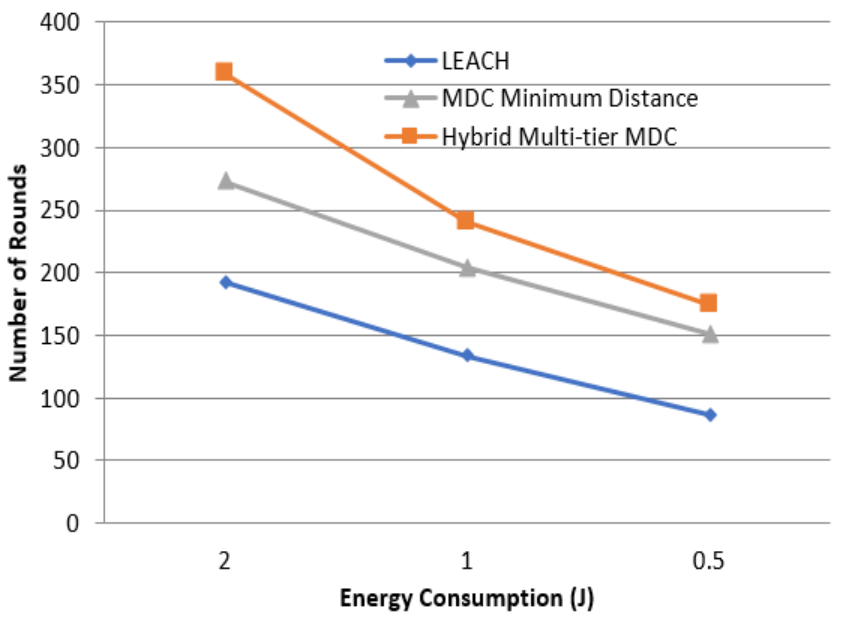

Fig. 2. First Node Die after 350 Rounds.

About 200 rounds (Not Sensor Nodes) including mobile and non-mobile are deployed in an open farming land to measure the performance of the network. Congregated results of simulation show a significant performance and high amount of data received in sample dense network. This experiment determines the energy consumed when the first node dies or has utilized $100 \%$ of its battery, maximum energy used when the last node dies, traffic received against node density, and total energy disbursed by nodes.

From the above Fig. 2 shows the state when first node consumed its $100 \%$ energy with compared to other mechanisms. It clearly observed that the presented scheme enhanced the node's lifetime and significantly consumed very less amount of energy with compare to LEACH and MDC minimum distance techniques. Where in LEACH, first node dies before the 200 rounds and utilized $1 / 4$ of energy in the earliest 90 rounds. On the other hand, in MDC minimum distance, first node relatively life longer than LECH and stays participating till 270 rounds and utilized $1 / 4$ of energy in the first 150 rounds. On contrary to $\mathrm{LEACH}$ and $\mathrm{MDC}$ minimum distance, Hybrid multi-tier MDC empower the nodes' health and prolong the its life that keeps it stay a life even after 350 rounds. Also, whereas LECH first node's consumed its 100\% energy and MDC minimum distance first node used its $50 \%$ of energy, the presented scheme used only $25 \%$ of its energy that lasts for early 180 rounds.

From the above Fig. 3, it can be observed that LEACH network dies in 800 rounds which has no match to proposed scheme. While MDC minimum distance network lived comparatively much longer than LECH and stays for about 2400 rounds and slightly energy curve depicts the irregular or sudden energy falls in some rounds that cost more energy. Whereas, the proposed scheme, significantly outperform other mechanisms and stays longer. The Hybrid Multi-tier MDC uses the consistence minimal amount of energy throughout the network that prolong the network life that showed by the straight decline energy slope. It stays a life for about 3500 rounds; which lives about 3000 rounds more than of LEACH and 1000 rounds more than of MDC minimum distance. 


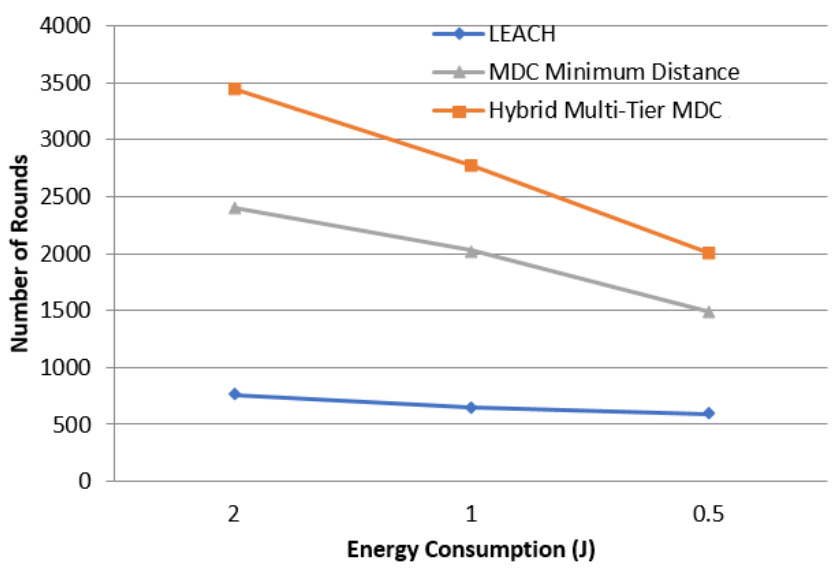

Fig. 3. Last node Compartive Status of LEACH, MDC Minimum Distance and Hybrid Multi-Tier MDC.

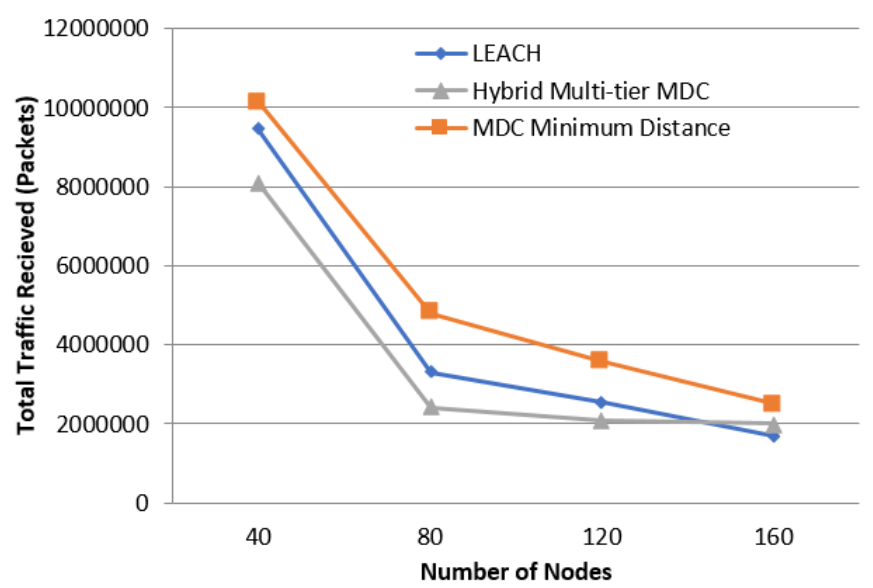

Fig. 4. Total packet Received in Dense Network Envirornment.

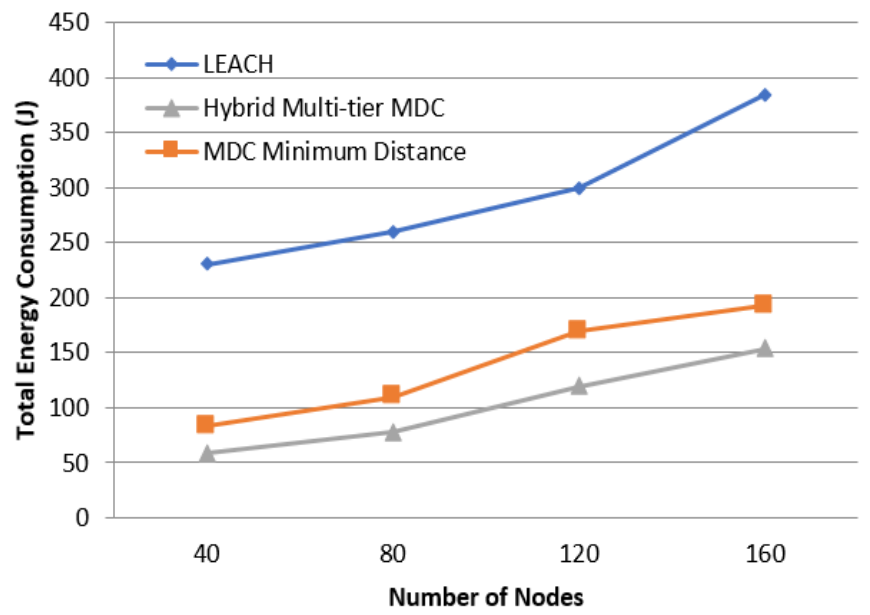

Fig. 5. Total Energy Consumption W.R.T Density.

The Fig. 4 shows the network traffic received in the presence of 40,80,120, and 120 nodes network environment. The result gradually shifting towards it favoritism from MDC minimum distance to our proposed scheme of Hybrid multitier MDC with the increasing density of network. In the above Fig., we can observed that by increasing the number of nodes or network density, the graph of other two schemes continuously decline while the proposed scheme graph getting stable and consistent with the density of network without losing any more network traffic packets in environment.

Beyond the individual node's life, the Fig. 5 represents total network energy utilization of said mechanisms with the increasing density of network. LEACH disbursed minimum $240 \mathrm{~J}$ energy in 40 nodes network and maximum about 400J energy in the presence of 160 nodes network. Also, rising LEACH graph reveals incompatibly of dealing with dense network. Although, there is negotiable energy shift in between 40 nodes to 120 nodes network that could be use as density trade off value. But, sudden rise in energy consumption after 120 make it unfavorable for dense network. On the other hand, MDC minimum distance graph shows comparatively better than LEACH. If we notice the graph, it used minimum 40J energy with 40 nodes and maximum 195J with the 160 nodes network. Besides this min and max energy utilization, the graph also tells an interesting fact that require some better scheme to overcome the gap that is the sudden rise in energy requirement after specific amount of node i.e. we can say after each 80 nodes. The energy utilization of between 40 to 80 and 120 to 160 nodes network can be deal as trade-off energy of node density, but the abrupt energy requirement rise right after 80 node. On contrary to both mechanisms, the proposed scheme showed much better results and consistency. The Hybrid multi-tier MDC graph represents its utilized minimum $50 \mathrm{~J}$ energy in 40 nodes network and maximum $150 \mathrm{~J}$ in 160 nodes network. Beyond the energy consumption index, also there is a gradual and consistence rise in energy need can be observed by the graph which can be deal as density trade-off i.e. rise in 4 times of nodes $(40 \times 4=160)$ as compare to minimum nodes amount rise only 3 times of energy need $(50 \times 3=150)$. Each add-ons of 40 nodes in network require only half amount of energy required by network.

\section{FUTURE WORK}

Due to financial constraints, the studied work is done under simulated environment with some planted huddles that shows provisioning results. Although, simulated work out perform the other exiting techniques, but it does not includes the real agriculture field impact on network life and cross field signal propagation huddles. In future, work will carry out in real agriculture filed to measure the proposed performance and gather more accurate results. Actual farm field experiment may unfolds the other parameters and better options to improve the communication scheme.

\section{CONCLUSION}

WSNs is a rapidly growing solution which is being adopted across a wide range of industries and businesses as well. Agricultural is a key domain in which WSNs offers remarkable solutions to eradicate agriculture field problems. In previous studies, we found two issues: the first is crossfield communication disturbance, and the second is early network dissolution. This paper uses the proposed MDC multi-tier technique to experiment and determine the network performance and lifetime in a dense open farmland. The artificial obstacles ingress in the simulated environment as cross- field obstacles with high number of nodes with respect to the size. Experimental results show a significant 
improvement when compared with prior methodologies. Presented MDC multi-tier approach prolongs the network lifecycle by saving individual node life due to which communication among the nodes becomes more reliable and only insignificant losses are measured in the presence of cross-field communication hurdles.

\section{REFERENCES}

[1] G. Dhand and S. S. Tyagi, "Data Aggregation Techniques in WSN:Survey," Procedia Comput. Sci., vol. 92, pp. 378-384, 2016.

[2] S. Jain, R. C. Shah, W. Brunette, G. Borriello, and S. Roy, "Exploiting mobility for energy efficient data collection in wireless sensor networks," Mob. Networks Appl., vol. 11, no. 3, pp. 327-339, 2006.

[3] P. Siripongwutikorn and B. Thipakorn, "Mobility-aware topology control in mobile ad hoc networks," Comput. Commun., vol. 31, no. 14, pp. 3521-3532, 2008.

[4] G. V Rama and L. V Srikanth, "Location-Based Routing Protocol in Wireless Sensor Network-A Survey," Int. J. Adv. Res. Comput. Sci. Softw. Eng., vol. 5, no. 4, 2015.

[5] P. S. Nithya Darisini and N. S. Kumari, "A survey of routing protocols for VANET in urban scenarios," Proc. 2013 Int. Conf. Pattern Recognition, Informatics Mob. Eng. PRIME 2013, pp. 464-467, 2013.

[6] S. Halder, P. P. Meta, and S. Banerjee, "MOBILITY AWARE ROUTING PROTOCOL IN AD-HOC NETWORK," CS IT, vol. 4, pp. 17-31, 2012.

[7] M. Arshad, N. Armi, N. Kamel, and N. M. Saad, "Mobile data collector based routing protocol for wireless sensor networks," Sci. Res. Essays, vol. 6, no. 29, pp. 6162-6175, 2011.

[8] Y. Khamayseh, G. Obiedat, and M. B. Yassin, "Mobility and Load aware Routing protocol for ad hoc networks," J. King Saud Univ. Comput. Inf. Sci., vol. 23, no. 2, pp. 105-113, Jul. 2011.

[9] A. Kumar, H. Y. Shwe, K. J. Wong, and P. H. J. Chong, "LocationBased Routing Protocols for Wireless Sensor Networks: A Survey," Wirel. Sens. Netw., vol. 9, no. 1, pp. 25-72, 2017.
[10] M. ARSHAD, M. Y. AALSALEM, and F. A. SIDDIQUI, "Energy efficient cluster head selection algorithm in mobile wireless sensor networks," J. Eng. Sci. Technol., vol. 9, no. 6, pp. 728-746, 2014.

[11] M. Di Francesco, S. K. Das, and G. Anastasi, "Data Collection in Wireless Sensor Networks with Mobile Elements: A Survey," ACM Trans. Sen. Netw., vol. 8, no. 1, p. 7:1-7:31, 2011.

[12] I. Mahgoub and M. Ilyas, Sensor Network Protocols. CRC Press, 2016.

[13] A. Norouzi, "An Integrative Comparison of Energy Efficient Routing Protocols in Wireless Sensor Network," Wirel. Sens. Netw., vol. 4, no. 3, pp. 65-75, 2012.

[14] A. Bokare, M. and Ralegaonkar, "Wireless Sensor Network," Int. J. Comput. Eng. Sci., vol. 2, no. 3, pp. 55-61, 2012.

[15] D. Goyal and M. R. Tripathy, "Routing Protocols in Wireless Sensor Networks: A Survey," 2012 Second Int. Conf. Adv. Comput. Commun. Technol., pp. 474-480, 2012.

[16] Muhammad Arshad, "Mobile data collector based routing protocol for wireless sensor networks," Sci. Res. Essays, vol. 6, no. 29, Nov. 2011.

[17] M. M. Chitlange, V. S. Deshpande, and S. Member, "Effect of node density on Congestion in WSN," Int. Conf. Pervasive Comput., vol. 0, no. c, pp. 4-6, 2015.

[18] F. A. Siddqui, M. Saeed, J. R. Khan, M. Arshad, and N. Touheed, "WSN Multi-Tier MDC GRID based Routing Protocol to mitigate Financial Impact," IJCSNS Int. J. Comput. Sci. Netw. Secur., vol. 17, no. 11, pp. 20-27, 2017.

[19] A. Salim, W. Osamy, and A. M. Khedr, "IBLEACH: Intra-balanced LEACH protocol for wireless sensor networks," Wirel. Networks, vol. 20, no. 6, pp. 1515-1525, 2014.

[20] P. K. VARSHNEY, G. S. AGRAWAL, and S. K. SHARMA, "Relative Performance Analysis of Proactive Routing Protocols in Wireless Ad hoc Networks using Varying Node Density," Invertis J. Sci. Technol., vol. 9, no. 3, pp. 1-9, 2016.

[21] D. Elmazi, M. Cuka, T. Oda, M. Ikeda, and L. Barolli, "Effect of Node Density on Actor Selection in WSANs : A Comparison Study for Two RE," in 31st International Conference on Advanced Information Networking and Applications Effect, 2017, pp. 865-871. 